\title{
ANESTHETIC MANAGEMENT OF CORNEAL TRANSPLANT IN A PATIENT WITH NON-BULLOUS CONGENITAL ICHTHYOSIFORM ERYTHRODERMA
}

\author{
D. Cancho, D. R. Zamudio, D. R. Delgado, G. Puebla, S. García del Valle, A. Rodríguez.
}

\section{INTRODUCTION}

The ichthyosis comprise a group of congenital disorders characterised by the presence of hyperkeratotic scales on the skin surface. Non-bullous congenital ichthyosiform erythroderma is an infrequent form of presentation, only few cases have been reported. This condition is inherited in an autosomal recessive pattern and the most common cause is inactivating mutations in the transglutaminase- 1 gene. Clinical manifestations include scaling of the skin, difficulty maintaining body temperature and in some cases malnutrition and musculoskeletal deformities.
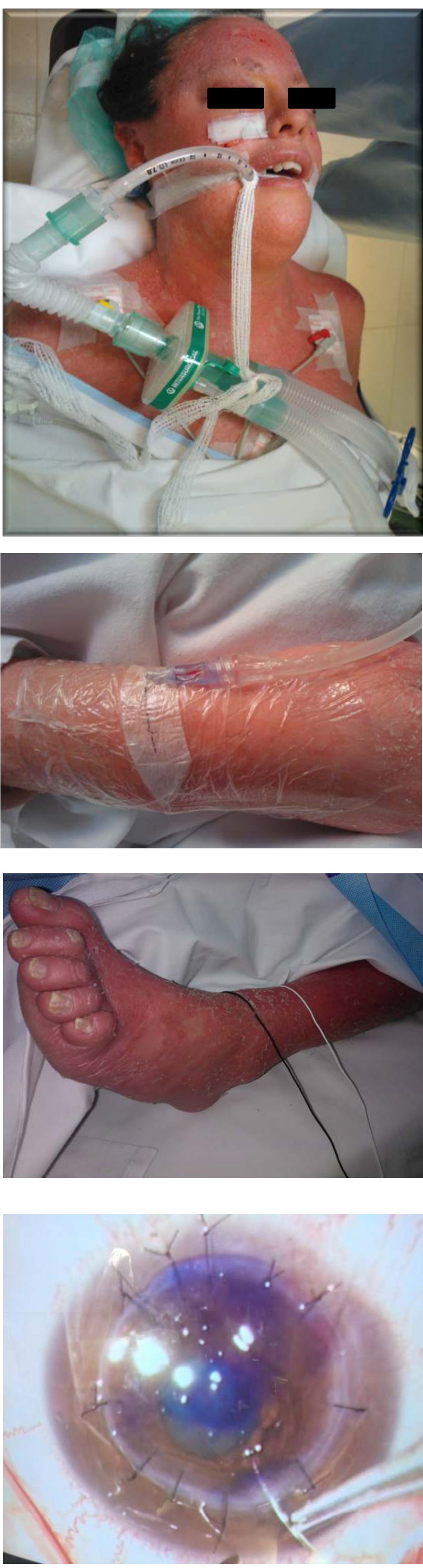

Hospital Universitario Fundación Alcorcón

\section{CASE REPORT}

We present the case of a 20 - year- old patient with non-bullous congenital ichthyosiform erythroderma who underwent corneal transplant under general anaesthesia. The patient presented erythroderma and generalised scaling of the whole integument, osteoarthritis, scoliosis, glaucoma and lagophthalmos. No difficult airway was anticipated. Intravenous access placement was difficult. Prior to induction of general anaesthesia we placed a $22 \mathrm{G}$ intravenous catheter and the patient received standard and neuromuscular monitoring. After induction, secure fixation of the endotracheal tube and intravenous cannula was performed, additionally proper positioning was revised and a forced-air warming system was used to maintain body temperature. Surgery was uneventful and the patient was extubated in the operating room without incidents.

\section{DISCUSSION}

* Because of scaling, fixation of the intravenous cannula, endotracheal tube and monitoring elements was difficult.

* Scaling also prevents from maintaining correct body temperature, consequently,temperature monitoring and body warmers were used. The skin in these type of patients is more sensitive to damage, thus, BIS monitoring was not placed but neuromuscular monitoring was used due to surgical requirements.

* Positioning and transportation was carefully performed considering musculoskeletal deformities.

* Venous access can be difficult because of abnormal keratinisation of the skin, which happened in our case.

\section{LEARNING POINTS}

- Difficult venous access.

* Difficult material fixation.

Risk of hypothermia.

- Difficult airway must be kept in mind because the progress of the disease may lead to restriction of mouth opening and neck mobility. 